\title{
Revisitation of the luminance conditions for the occurrence of the achromatic neon color spreading illusion
}

\author{
PAOLA BRESSAN \\ University of Padua, Padua, Italy
}

\begin{abstract}
This paper develops the idea (Bressan, 1993) that neon spreading derives from the perceptual scissioning of ordinary assimilation color, a process identical to that occurring with nonillusory colors in phenomenal transparency. It is commonly held that the critical elements in achromatic neon spreading patterns must be of luminance intermediate between that of the embedding lines and of the background. The interpretation of neon spreading on the basis of color scissioning, however, predicts that neon spreading should also be observed for different luminance hierarchies, provided that these are compatible with transparency. This prediction found experimental support in the present work. The results suggest that (1) the widespread notion that chromatic and achromatic neon spreading must be mediated by separate mechanisms is unwarranted; (2) the widespread notion that color spreading in ordinary assimilation patterns and color spreading in neon patterns must be mediated by separate mechanisms is unwarranted; and (3) other than pointing to the way in which the overall organization of a scene affects the mode of color appearance, the neon spreading effect may not convey any extra theoretical relevance.
\end{abstract}

The neon color spreading illusion (van Tuijl, 1975; Varin, 1971) occurs when, in a line drawing, sections of the lines are replaced by segments of a different (chromatic or achromatic) color. This color then appears to spread out of the segments, giving rise to the perception of a slightly tinted transparent surface floating above the pattern; alternatively, the observer has the impression of a unitary, uniformly colored lattice of lines, onto which a differently colored light (or shadow) is cast by an independent outside source.

It is commonly held that neon color spreading takes place only if certain figural and luminance conditions occur together. The classic figural prerequisites are the presence of subjective contours, connection and collinearity between the segments and the lines in which they are embedded, and similar stroke widths (Redies \& Spillmann, 1981). At the same time, the luminance of the segments must be between the luminances of the external (embedding) lines and of the background (van Tuijl \& de Weert, 1979). If these conditions are not satisfied, no neon effect can be perceived; instead, ordinary spreading due to Bezold-type assimilation may be observed, lacking the defining features of neon spreading (van Tuijl \& de Weert, 1979; van Tuijl \& Leeuwenberg, 1979). In this case, the pattern is not perceived as one unitary line structure, and the difference between the illusorily colored area and its surround is not perceived as due to a veil or a light; rather, one has the impression of two coplanar regions of different colors. On the basis of both the different conditions

Correspondence should be addressed to P. Bressan, Dipartimento di Psicologia Generale, Università di Padova, Piazza Capitaniato 3, 35139 Padova, Italy (e-mail: bressan@unipad unipd.it). for their occurrence and their different phenomenal nature, neon color spreading and classic assimilation have been regarded as two separate and unrelated perceptual phenomena.

Bressan (1993) has recently shown that the figural conditions for neon spreading can be violated as long as perceptual transparency is maintained. On the basis of this and other observations, she argued that neon spreading and assimilation share the same low-level mechanism, and that the special sensory conditions for the occurrence of neon spreading are in fact the sensory conditions for the occurrence of perceptual transparency. As for the diversity between neon spreading and assimilation from a phenomenological standpoint, Bressan proposes that neon spreading is to assimilation as transparent, see-through color is to surface color. In other words, the single, key difference between neon spreading and Bezold-type spreading would be the presence or absence of perceptual scissioning of the illusory color from the plane of the figure. The one important feature that all neon spreading patterns share is that the background color is separated from the illusory color and, instead of mixing with it, is seen through it. Bressan suggests that Bezold-type assimilation phenomenally turns into the neon spreading effect whenever (i.e., if and only if) it takes place within a surface that is also seen as transparent. The illusory tinge, then, rather than merging with the solid background color, becomes a translucent layer, taking on the "vagueness" and the typical floating, rarefied quality of the neon effect. ${ }^{1}$

For this idea to be accepted, the implication must be shown to hold in both directions: first, any assimilationtinted surface that gives rise to a neon spreading effect ought to appear transparent; second, any assimilation- 
tinted surface that appears transparent ought to give rise to a neon spreading effect. As to the first point, as far as I know, all existing descriptions of neon spreading involve mention of phenomenal transparency (in terms of either a veil or a light). (The issue of transparency perception's being a necessary condition for the emergence of neon color spreading has been addressed directly by Meyer \& Dougherty, 1987, and Nakayama, Shimojo, \& Ramachandran, 1990; and indirectly by de Weert \& van Kruysbergen, 1987.)

But what about the other side of the implication? It obviously entails that the luminance conditions for neon spreading cannot be stricter than the luminance conditions for transparency. The luminance conditions for the achromatic version of the neon spreading effect have been mentioned above: the target lines must be of luminance intermediate between that of the external lines and that of the background for a neon effect to become possible (van Tuijl $\&$ de Weert, 1979). No illustrations of the neon spreading phenomenon for the sake of either demonstration or research have so far prescinded from this basic rule. Several authors (e.g., Nakayama et al., 1990) have in fact stressed that this luminance hierarchy corresponds to that for transparency, reached when the object that is to be perceived as transparent is of luminance intermediate between that of the occluded object and that of the background (Metelli, 1974b).

Unfortunately, there is a problem here. Although it is true that in the most often reprinted illustrations of phenomenal transparency-like that in Figure 1-both the splitting regions $P$ and $Q$ have luminances intermediate between those of the underlying regions $A$ and $B$ (that is, $a>p>q>b$ or $a<p<q<b$ ), these cases are by no means the only ones described by Metelli and theoretically allowed by his equations. For instance, Metelli (1974a) reported the case $a>p>b>q$, which does not conflict with the necessary conditions for transparency and gives rise to a dark transparent layer. Here,

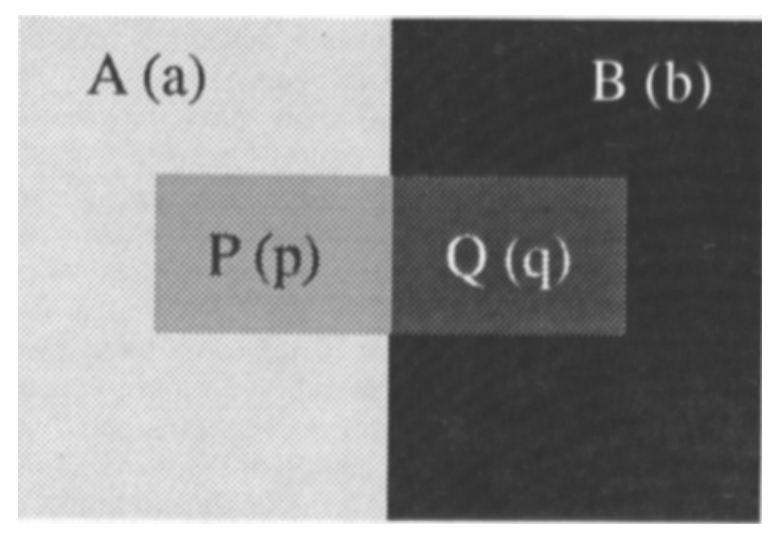

Figure 1. A typical four-surface configuration that gives rise to the impression of a transparent filter in front of a bicolored background. Capital letters indicate regions of different intensity; lowercase letters indicate the corresponding lightnesses. one of the splitting regions $(\mathrm{Q})$ is the darkest. Obviously, the reverse case $a<p<b<q$, where the splitting region $Q$ is the lightest, will give rise to a light transparent layer. The problem, then, is that this family of cases does not comply with van Tuijl and de Weert's (1979) conditions for the occurrence of neon spreading, and this represents a strong argument against the thesis that neon spreading and transparency are intimately related. In this paper I wish to address this point.

\section{The Lightness Conditions for the Occurrence of Transparency}

Although his transparency equations had been originally expressed in terms of reflectances, Metelli later accepted Beck, Prazdny, and Ivry's (1984) suggestion that they should be expressed in terms of lightnesses (Metelli, 1985). In the following, I will refer mostly to lightnesses, except when handling luminances will seem more appropriate. Only the case of achromatic color spreading will be dealt with in this paper.

Metelli's (1985) first lightness condition of transparency can be expressed as follows:

$$
(a>b) \Leftrightarrow(p>q)
$$

and

$$
(a<b) \Leftrightarrow(p<q),
$$

where " $>$ " means lighter and " <" means darker. That is, the lightness gradients between A and B and between $P$ and $Q$ must have the same direction; in other words, the lighter (darker) region of a transparent overlay must lay on the lighter (darker) region of the background. This is an order restriction; it reflects the physical fact that a uniform transparent filter preserves, and cannot invert, the order of intensities across the underlying areas.

Metelli's second lightness condition for transparency can be expressed as follows:

$$
|a-b|>|p-q| \text {. }
$$

That is, the difference between the lightnesses of the regions $A$ and $B$ must be larger than the difference between the lightnesses of the regions $\mathbf{P}$ and $\mathbf{Q}$. This is a magnitude restriction; it reflects the physical fact that a uniform transparent filter reduces, and cannot increase, the intensity difference between the underlying areas.

Although physical transparency is impossible if either condition is infringed, Beck and Ivry (1988) found that, for a certain class of violations, perceived transparency was still reported. In general, the frequency of transparency judgments tended to vary inversely with the magnitude of the violation. These authors presented evidence for two kinds of violations of the order and magnitude restrictions: simple, for which transparency judgments, while numerically reduced, still occurred, and strong, for which transparency judgments were virtually absent.

A simple violation of the order restriction violates only the first lightness condition for transparency. A strong violation also violates the following: 


$$
(a>p) \Leftrightarrow(b>q)
$$

and

$$
(a<p) \Leftrightarrow(b<q) .
$$

A strong violation thus means that the transparent overlay inverts not only the order of intensities across the underlying areas, but also the order of intensities across its own boundary.

A simple violation of the magnitude restriction violates only the second lightness condition for transparency. A strong violation occurs when the lightnesses of the regions $\mathrm{A}$ and $\mathrm{B}$ are contained within the lightness interval defined by the regions $\mathrm{P}$ and $\mathrm{Q}$-in other words, when $p$ and $q$ are at the extremes of the lightness hierarchy. A strong violation thus means that the transparent overlay, in addition to increasing the intensity difference between the underlying areas, inverts the order of intensities across its own boundary.

Also notice that the basic equation of transparency,

$$
\alpha=p-q / a-b,
$$

implies that when the difference between $a$ and $b$ is much larger than the difference between $p$ and $q$, the perceived degree of transparency $\alpha$ will be low, whereas when the difference between $a$ and $b$ is only slightly larger than the difference between $p$ and $q$, the perceived degree of transparency will be high.

\section{Prediction of the Lightness Conditions for the Occurrence of Neon Color Spreading}

Figure 2 shows a classic neon spreading configuration: the modified Ehrenstein figure (first used by Redies \& Spillmann, 1981). Let $a, b, p$, and $q$ be, respectively, the

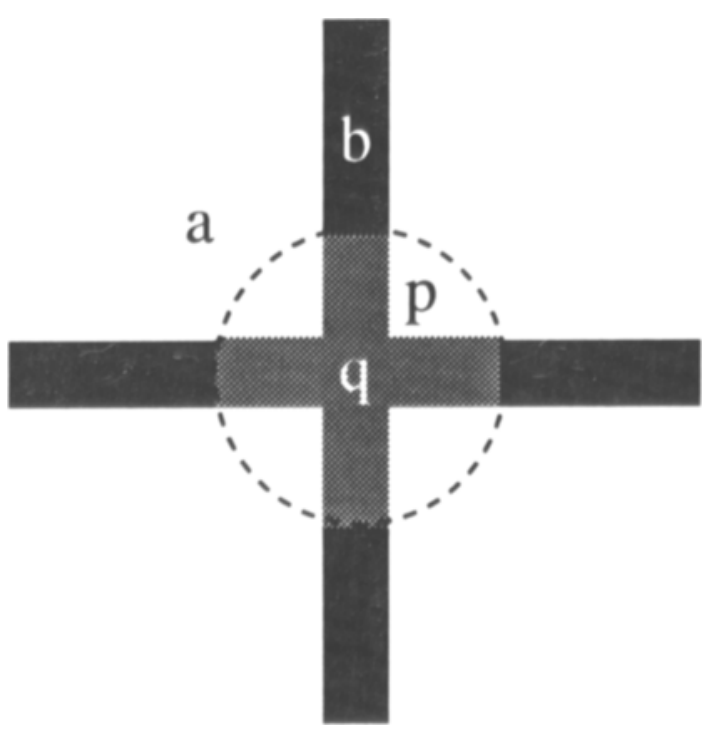

Figure 2. A typical neon spreading pattern (the modified Ehrenstein figure) interpreted as a four-surface transparency configuration; $p$ represents the illusory color. lightnesses of the background (Region A), of the external lines (Region B), of the region on which assimilation takes place (Region $P$, usually corresponding to the subjective figure), and of the target lines (Region Q). Notice that $p$, being the assimilation color, is always between $a$ and $q$ (and very close to $a$ ) when it comes to lightness. As can be seen in Figure 2, $a>p>q>b$; the conditions for transparency are fulfilled and the critical area color $p$ will split into a background component identical to the color of the surround A (the background will therefore appear homogeneous everywhere) and a foreground component corresponding to the color shift (the phenomenally transparent overlay represented as a dotted circle in Figure 2).

Since only three, rather than four, luminance values can be manipulated in neon spreading figures (i.e., those corresponding to the regions $A, B$, and $Q$ ), only six separate lightness sequences are possible. As each sequence and its reverse are equivalent (corresponding to either direction of contrast), three different cases follow. ${ }^{2}$

Case 1. The target lines $\mathrm{Q}$ are intermediate in lightness between the external lines $B$ and the background $A$ (lightness hierarchy $a>p>q>b$ or $a<p<q<$ $b$ ). In this case, the magnitude restriction $|a-b|>|p-q|$ is always fulfilled. In fact, the background $A$ and the external lines $B$ assume extreme values (typically, white and black, or vice versa). The order restrictions $(a>b) \Leftrightarrow$ $(p>q)$ (for positive figure-ground contrast) and $(a<b$ ) $\Leftrightarrow(p<q)$ (for negative figure-ground contrast) are also always fulfilled. It follows that transparency is theoretically allowed (and maximally probable) for all possible values of $a, b$, and $q$. This is the case represented in Figure 2, and it corresponds to van Tuijl and de Weert's (1979) conditions for the occurrence of neon spreading.

Case 2. The background $A$ is intermediate in lightness between the external lines B and the target lines $Q$ (lightness hierarchy $q>p>a>b$ or $q<p<a<b$ ). In this case, the magnitude restriction $|a-b|>|p-q|$ can be fulfilled or not depending on the actual values of its terms. (More specifically, given that $p$ is very close to $a$, this expression will be true if the intermediate color $a$ is closer to the color of the target lines $q$ than to the color of the external lines $b$; it will be false in the opposite case.) On the other hand, the order restrictions $(a>b)$ $\Leftrightarrow(p>q)$ (for positive figure-ground contrast) and $(a<b) \Leftrightarrow(p<q)$ (for negative figure-ground contrast) are never met. In fact, if the target region $Q$ is the lightest, it is $q>p>a>b$; but the order restriction of transparency states that $a>b$ must imply $p>q$, which is false since $q>p$. If the target region $\mathrm{Q}$ is the darkest, it is $q<p<a<b$; but the order restriction states that $a<b$ must imply $p<q$, which is false since $q<p$.

On the other hand, because $p$ is an assimilation color, the contrast across the B-Q boundary and the contrast across the A-P boundary have necessarily the same sign. The transparent overlay thus never inverts the order of intensities across its own boundary; therefore, violations 
of the order and magnitude restrictions are always simple violations.

It follows that Case 2 always represents a simple violation of the order restriction (the closer $p$ and $q$ are, the less severe this violation will be) and may additionally represent a simple violation of the magnitude restriction, depending on the actual values of $a, b$, and $q$.

Case 3. The external lines B are intermediate in lightness between the background $\mathrm{A}$ and the target lines $\mathrm{Q}$ (lightness hierarchy $q>b>p>a$ or $q<b<$ $p<a)$. In this case, the order restrictions $(a>b) \Leftrightarrow$ $(p>q)$ (for positive figure-ground contrast) and $(a<b$ ) $\Leftrightarrow(p<q)$ (for negative figure-ground contrast) are always respected. The magnitude restriction $|a-b|>$ $|p-q|$, however, is only fulfilled when $|b-q|<|a-p|$. Since $a$ and $p$ are very close (remember that the corresponding luminances are identical), this can only happen when the difference between $b$ and $q$ is extremely small. On the other hand, since the contrast across the B- $Q$ boundary and the contrast across the A-P boundary have the same sign, the violation of the magnitude restriction is a simple violation.

It follows that Case 3 virtually always represents a simple violation of the magnitude restriction; the closer $b$ and $q$ are, the less severe this violation will be.

From the preceding analysis, a specific prediction follows: if the emergence of neon spreading depends on the occurrence of phenomenal transparency, neon spreading should also be observed for luminance conditions different from those reported by van Tuijl and de Weert (1979). In particular, it should be observed when the external lines $B$ are intermediate in luminance between the background $A$ and the target lines $Q$ (Case 3), provided that the lu- minance of the target lines is as close as possible to the luminance of the external lines. Since in this case the difference between $|a-b|$ and $|p-q|$ will be small, it is also predicted that for this class of stimuli the neon-like layer will appear to have a high degree of transparency (i.e., little density). As the difference between the lightnesses of the target and the external lines increases, the probability of neon spreading should accordingly decrease. Some effect may also be observed when the background $\mathrm{A}$ is intermediate in luminance between the external lines $\mathrm{B}$ and the target lines $\mathrm{Q}$ (Case 2), provided that the luminance of the target lines is as close as possible to the luminance of the background.

\section{EXPERIMENT 1}

\section{Luminance Conditions for Neon Spreading Patterns With Intermediate-Luminance Backgrounds}

\section{Method}

The stimuli consisted of eight Varin-type figures like those shown in Figure 3; the sides of the subjective squares were $25 \mathrm{~mm}$. The four pictures in the top row represent four separate instances of the sequence $q>p>a>b$; the four pictures in the bottom row represent the equivalent instances of its reverse $q<p<a<b$ (Case 2). Five different shades of gray (60-line-per-in dot patterns) were used, corresponding to the following five black/white ratios: $0 \%$ (white), $25 \%$ (light gray), 50\% (mid gray), $75 \%$ (dark gray), and $100 \%$ (black). In each picture, the background was always $50 \%$ gray.

All the patterns violate the order restriction, since the lighter of the overlaying regions is always over the darker of the underlying regions and vice versa. However, as I have shown above, the magnitude of this violation varies directly with the difference between $p$ (the lightness of the square region) and $q$ (the lightness of the target lines). Therefore, Patterns $b$ and $d$ represent less severe vio-

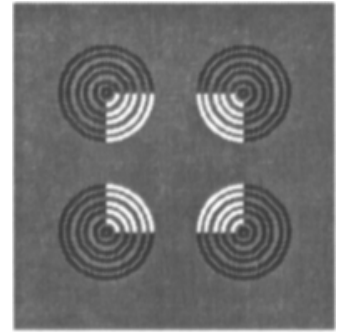

$a^{\prime}$

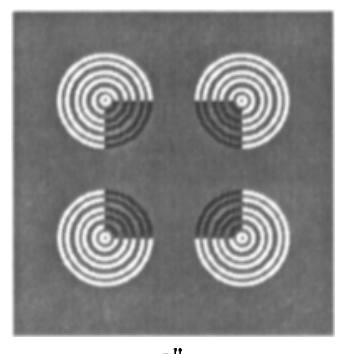

$\mathbf{a}^{\prime \prime}$

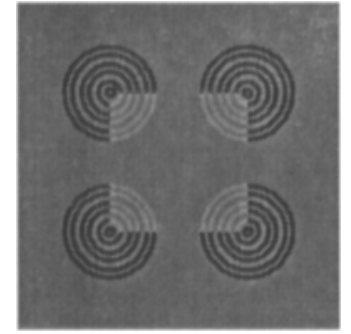

$b^{\prime}$

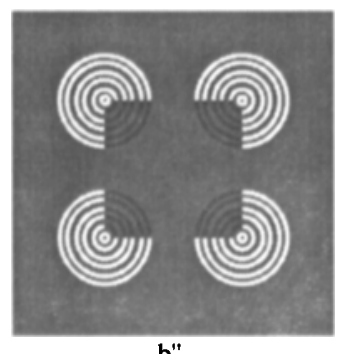

$\mathbf{b}^{\prime \prime}$

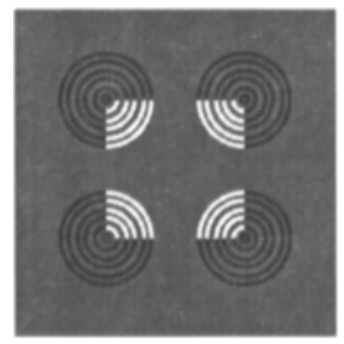

$\mathbf{c}^{\prime}$

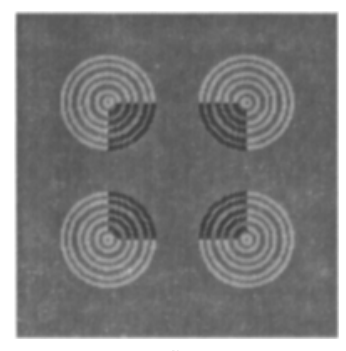

$c^{\prime \prime}$

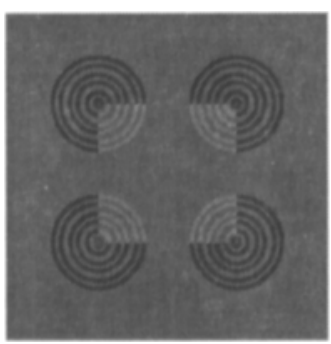

$\mathbf{d}^{\prime}$

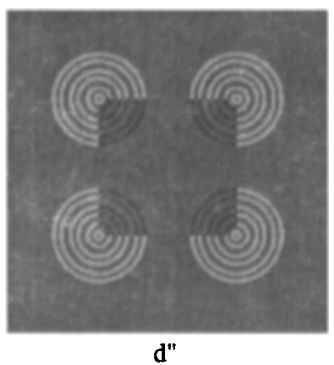

Figure 3. The patterns used in Experiment 1. The luminances of the figures shown here are representative of those used in the stimuli but do not reproduce them exactly. 
Iations than do Patterns a and c. (Patterns $d$ may represent an even slighter violation than do Patterns $b$, because the difference between $a$ and $b$ is smaller.) Patterns $c$ additionally violate the magnitude restriction, since the difference between the lightnesses of the overlaying regions (target lines and square) is larger than the difference between the lightnesses of the underlying regions (external lines and background).

The figures were presented one at a time in a random order, from a distance of about $60 \mathrm{~cm}$. Five experienced subjects took part in the experiment. (The stimulus figures of all the experiments reported in this paper were also informally shown to a number of naive observers; the pattern of results was the same.) A pilot experiment had shown that the possible percepts corresponding to this type of stimuli are four: (1) a transparent square filter in front of four concentric-circle patterns lying on the background; (2) a square patch of light or shade cast upon four concentric-circle patterns lying on the background; (3) an opaque square figure partly covering four concentric-circle patterns lying on the background (in which case the target lines are pasted on the square); and (4) a large transparent area with a central square hole (in which case the target lines sit on the background and are seen through the hole).

The subjects were asked to observe each picture, to describe it thoroughly, and then, to assign it to one of the four categories listed above. Whenever the percept was ambiguous, they were requested to look at the picture for a couple of minutes and to report which of the alternative percepts was the most stable

\section{Results}

Judgments of neon spreading were based on the simultaneous presence of the following features: (1) four figurally unitary and thoroughly visible concentric-circle patterns; (2) a square region of a different lightness from the background; (3) the impression that this region was either a transparent filter or a patch of light (or shadow) cast by an independent outside source.

Note that these features are the only ones shared by all neon spreading cases reported in the literature; Features 1 and 3 are typical of the neon spreading phenomenon and differentiate it from Bezold-type assimilation. Neonlike luminescence was not included in the list, because it should only be considered as an incidental property of some neon spreading patterns (see Bressan, 1993); it is enough to think that it is never observed in neon spreading figures with negative figure-ground contrast.

Table 1 shows the frequency of the alternative percepts. Neon color spreading was elicited easily by Patterns $b$ and Patterns d, always in terms of a transparent filter rather than of a light. The probability of neon spreading decreased steeply for Patterns a and Patterns c, where the front-frame and front-square percepts were both possible; in general, the frame was reported almost twice as frequently as the square.

In conclusion, neon color spreading was observed in patterns where the background luminance was intermediate between that of the external lines and that of the target lines, provided that the luminance of the target lines was as close as possible to the luminance of the background. When this condition was not met, neon color spreading tended to be replaced by ordinary color assimilation, occurring within an opaque region.
Table 1

Response Frequencies (in Percentages) to Stimuli With Intermediate-Luminance Backgrounds

\begin{tabular}{|c|c|c|c|c|c|}
\hline \multirow[b]{2}{*}{ Pattern } & \multicolumn{2}{|c|}{ Assimilation } & \multicolumn{3}{|c|}{ Neon Spreading } \\
\hline & $\begin{array}{l}\text { Front } \\
\text { Frame }\end{array}$ & $\begin{array}{l}\text { Front } \\
\text { Square }\end{array}$ & $\begin{array}{c}\text { Light/ } \\
\text { Shadow }\end{array}$ & Filter & Total \\
\hline$a^{\prime}$ & 60 & 20 & 0 & 20 & 20 \\
\hline a" & 20 & 40 & 0 & 40 & 40 \\
\hline$b^{\prime}$ & 0 & 0 & 0 & 100 & 100 \\
\hline$b^{\prime \prime}$ & 0 & 20 & 0 & 80 & 80 \\
\hline$c^{\prime}$ & 60 & 0 & 0 & 40 & 40 \\
\hline$c^{\prime \prime}$ & 60 & 40 & 0 & 0 & 0 \\
\hline$d^{\prime}$ & 0 & 0 & 0 & 100 & 100 \\
\hline $\mathrm{d}^{\prime \prime}$ & 0 & 0 & 0 & 100 & 100 \\
\hline
\end{tabular}

\section{EXPERIMENT 2}

\section{Luminance Conditions for Neon Spreading Patterns With Intermediate-Luminance External Lines}

\section{Method}

The stimuli consisted of six Varin-type figures (shown in Figure 4) similar in all respects to those of Experiment 1, except for the luminance hierarchy. The three pictures in the top row represent four separate instances of the sequence $q>b>p>a$; the three pictures in the bottom row represent the equivalent instances of its reverse $q<b<p<a$ (Case 3). In the pictures at the top, the background was always black $(100 \%)$ and the target lines white $(0 \%)$; the external lines were light gray $\left(25 \%, a^{\prime}\right)$, medjum gray $\left(50 \%, b^{\prime}\right)$, and dark gray $\left(75 \%, c^{\prime}\right)$. In the pictures at the bottom, the background was always white $(0 \%)$ and the target lines black $(100 \%)$; the external lines were medium gray $\left(50 \%, a^{\prime \prime}\right)$, light gray $\left(25 \%, b^{\prime \prime}\right)$, and very light gray $\left(10 \%, c^{\prime \prime}\right)$. (Lighter grays had to be used in the bottom row because of the considerable perceptual darkening they underwent because of simultaneous contrast with the white background.)

All these figures violate the magnitude restriction, since the difference between the lightnesses of the overlaying regions (target lines and square) is always larger than the difference between the lightnesses of the underlying regions (external lines and background). The closer target and external lines are in lightness, however, the slighter this violation will be. Therefore Patterns a, b. and $\mathrm{c}$ represent increasingly severe violations.

Five experienced subjects (the same who had taken part in the previous experiment) acted as observers. The procedure was identical to that used in Experiment 1.

\section{Results}

Table 2 shows the frequency of the alternative percepts. Neon color spreading was unanimously perceived in Patterns a. The probability of neon spreading decreased gradually for Patterns b and Patterns $c$, where neon spreading in the central square was replaced by the impression of an opaque background seen through the central hole of a semitransparent frame. Overall, the illusory color was attributed to a cast light (or shadow) as frequently as to a superimposed filter, but the direction of contrast (top- vs. bottom-row pictures) was not ineffectual. For the white target lines, about $80 \%$ of the times the illusory square was seen as a spot of light rather than as a light filter; for the black target lines, about $80 \%$ of 


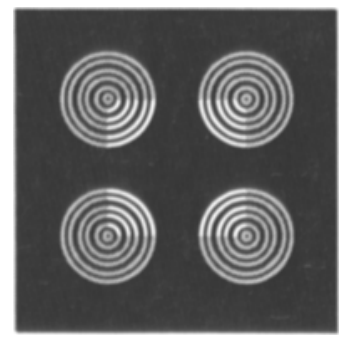

$\mathbf{a}^{\prime}$

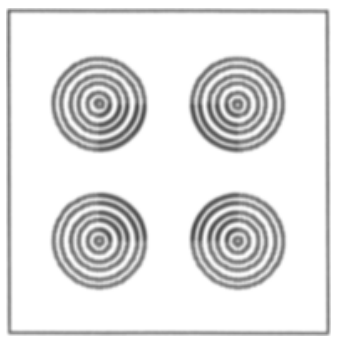

$\mathbf{a}^{\prime \prime}$

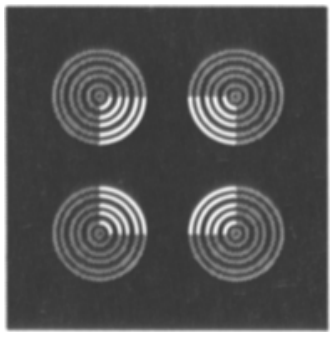

$b^{\prime}$

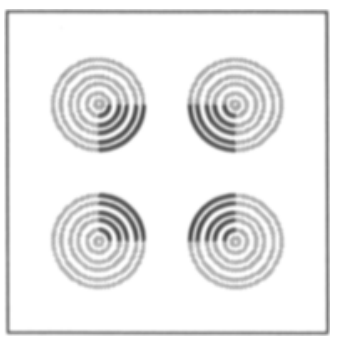

b"

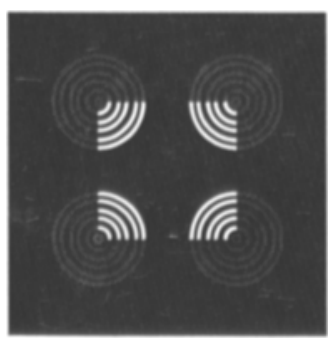

$c^{\prime}$

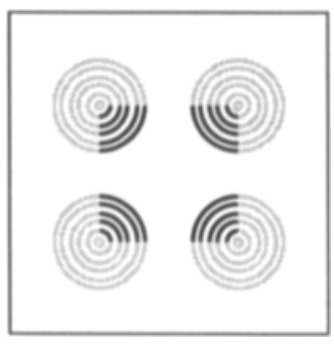

$c^{\prime \prime}$

Figure 4. The patterns used in Experiment 2. The luminances of the figures shown here are representative of those used in the stimuli but do not reproduce them exactly.

the times the illusory square was seen as a dark filter rather than as a cast shadow.

In conclusion, neon color spreading was easily observed in patterns where the external lines were intermediate in luminance between the target lines and the background. Only when the luminances of the target and external lines were very far apart was neon color spreading replaced by ordinary color assimilation, occurring within an opaque region.

\section{EXPERIMENT 3 \\ Phenomenal Attributes of Neon Spreading Patterns Depend on the Luminance Hierarchy}

\section{Method}

The stimuli consisted of six Varin-type figures, each implementing a different relation between $a, b, p$, and $q$ (see Figure 5). Stimulus la represented the sequence $a>p>q>b$, Stimulus $1 \mathrm{~b}$ its reverse $a<p<q<b$ (Case 1); Stimulus 2a represented the sequence $q>p>a>b$, Stimulus $2 \mathrm{~b}$ its reverse $q<p<a<b$ (Case 2); Stimulus 3a represented the sequence $q>b>p>a$,

Table 2

Response Frequencies (in Percentages) to Stimuli With Intermediate-Luminance External Lines

\begin{tabular}{|c|c|c|c|c|c|}
\hline \multirow[b]{2}{*}{ Pattern } & \multicolumn{2}{|c|}{ Assimilation } & \multicolumn{3}{|c|}{ Neon Spreading } \\
\hline & $\begin{array}{l}\text { Front } \\
\text { Frame }\end{array}$ & $\begin{array}{l}\text { Front } \\
\text { Square }\end{array}$ & $\begin{array}{l}\text { Light/ } \\
\text { Shadow }\end{array}$ & Filter & Total \\
\hline $\mathbf{a}^{\prime}$ & 0 & 0 & 80 & 20 & 100 \\
\hline$a^{\prime \prime}$ & 0 & 0 & 40 & 60 & 100 \\
\hline $\mathbf{b}^{\prime}$ & 40 & 0 & 60 & 0 & 60 \\
\hline$b^{\prime \prime}$ & 40 & 0 & 0 & 60 & 60 \\
\hline $\mathrm{c}^{\prime}$ & 80 & 0 & 0 & 20 & 20 \\
\hline$c^{\prime \prime}$ & 80 & 0 & 0 & 20 & 20 \\
\hline
\end{tabular}

Stimulus $3 b$ its reverse $q<b<p<a$ (Case 3). For Cases 2 and 3 , the actual luminance values were chosen among those that, in the previous experiments, had been shown to give the best neon spreading effect.

The figures were presented together, from a distance of about $60 \mathrm{~cm}$. Five experienced subjects (the same who had taken part in Experiments 1 and 2) acted as observers. They were asked to describe and compare the pictures on the basis of their more salient phenomenal attributes. In particular, they had to report on the following features: location in depth of the altered-color square relative to the background and to the concentric patterns; degree of transparency (on a 10-point scale) of the central square; uniformity of transparency within the square; color of the square; uniformity of color within the square; and color constancy. Color constancy (see Beck \& Ivry, 1988) was defined in terms of whether the color of the part of the lattice seen through the transparent layer (corresponding to the target lines) looked the same as the color of the part of the lattice seen directly (external lines). In other words, it referred to the chromatic continuity of the concentric patterns. This feature was not included among the defining attributes of neon spreading, because the issue of whether chromatic continuity of the underlying structure should be regarded as an essential characteristic of the effect has never been specifically addressed.

\section{Results}

Neon color spreading was reported for all patterns. Each filter was seen as homogeneous in color and degree of transparency and as appearing in front of uniformly colored concentric-circle patterns. In other words, transparency was always balanced and color constancy was preserved. In Case 3 patterns, the illusory square tended to appear in front of the concentric circles when it was seen as a filter, but it could appear behind them when it was seen as a patch of light or shade.

On the average, the filter looked white in 1a, dark gray in $1 \mathrm{~b}$; light gray in $2 \mathrm{a}$, medium gray in $2 \mathrm{~b}$; and luminous, 


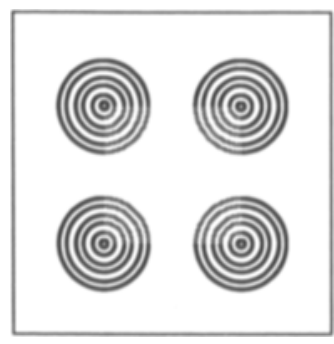

la

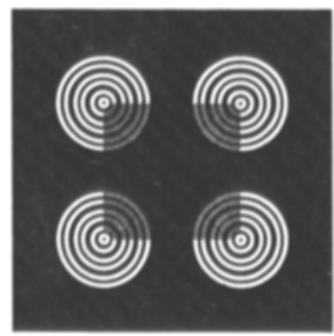

$1 \mathbf{b}$

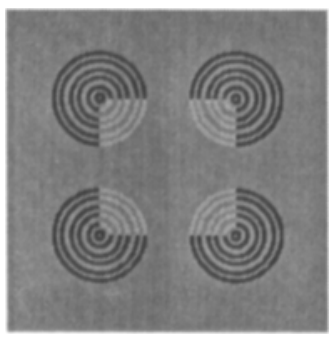

$2 \mathrm{a}$

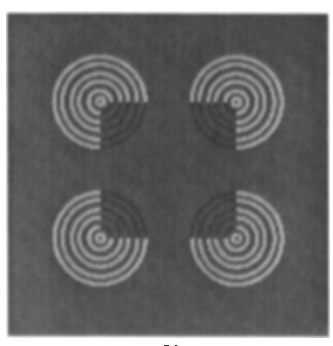

$2 b$

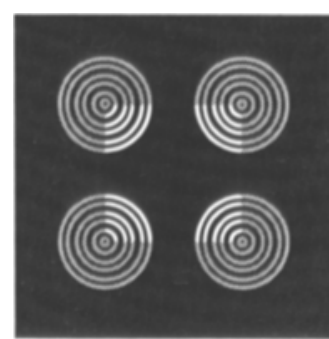

$3 \mathbf{a}$

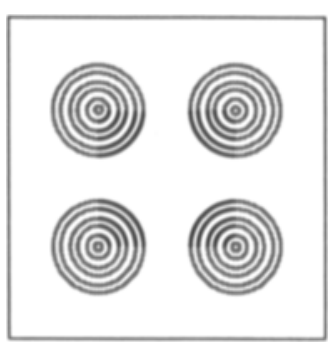

$3 b$

Figure 5. The patterns used in Experiment 3. The luminances of the figures shown here are representative of those used in the stimuli but do not reproduce them exactly.

but not colored, in $3 \mathrm{a}$ and light gray in $3 \mathrm{~b}$. Transparency was judged on a scale ranging from 0 (opaque) to 10 (perfectly transparent). The degree of transparency of the filters was found to depend both on the lightness sequence (averaging respectively $3,5.1$, and 8.6, for Cases 2,1 , and 3 ) and on the direction of contrast (being always higher for light target lines). The highest perceived transparency was reported for Case 3 ( $3 \mathrm{a}, M=9.2 ; 3 \mathrm{~b}, M=$ 8.0); the transparency of Case 1 patterns was intermediate $(1 \mathrm{a}, M=6.2 ; 1 \mathrm{~b}, M=4.0)$; the transparency of Case 2 patterns was the lowest $(2 \mathrm{a}, M=3.6 ; 2 \mathrm{~b}, M=$ 2.4). Occasionally, Case 1 and 2 patterns were described as "milky" or "misty"; Case 1a, 2a, and 3a patterns (top row) were described as slightly luminescent.

\section{DISCUSSION}

The experiments reported here show that neon color spreading is observed in luminance conditions different from those reported by van Tuijl and de Weert (1979); in fact, it occurs with any possible luminance hierarchy, provided that there is an impression of overlapping colors. I can see at least two separate reasons why these cases might have been discarded originally. In van Tuijl and de Weert's experiment, judgments of neon spreading were based on the simultaneous presence of three features: (1) a unitary lattice continuing underneath a superimposed figure; (2) embeddedness of the target structure; and (3) vagueness of the target lines as opposed to the external lines. It is clear that when the luminance of the target lines is the highest (on a dark background) or the lowest (on a light background), Condition 3 cannot possibly be met. Similarly, Condition 3 will not be satisfied when the luminances of target and external lines deviate in opposite directions, and by the same amount (as in their stimulus patterns), from the background. In other words, Condition 3 per se confines judgments of neon spreading to patterns where the target lines are less contrastive than the external lines, since only in this case can the former be considered more "vague."

The second reason stirs up a new issue. If the assimilation color is there anyway, and if the neon spreading effect becomes apparent only when transparency is seen, we may expect that figural factors favoring or hindering transparency will play a role. It has been shown (Beck et al., 1984) that Metelli's luminance conditions tolerate tougher violations when figural cues suggest overlying surfaces than when they do not. From the figural organization viewpoint, stimuli like those depicted in Figures 3,4 , and 5 are different from the lattices used by van Tuijl and de Weert (1979). Varin-type patterns suggest interposition so strongly that a subjective figure is seen anyway, whether as a square or as a frame. On the other hand, possibly because of their highly regular and iterative nature, van Tuijl-type patterns may sometimes be perceived simply as lattices where some segments are lighter or darker than the others. Neon spreading will not be reported in these cases; but transparency will not be reported either. This issue is of some importance, since it implies that the results of studies done on remarkably different neon spreading patterns (typically, the modified Ehrenstein pattern, van Tuijl-type lattices, and Varin-type figures) might not be comparable.

The finding that neon spreading can occur in a wide range of luminance conditions has another important consequence. It is widely believed (see, e.g., Ejima, Redies, 
Takahashi, \& Akita, 1984) that neon brightness and neon color spreading - that is, the achromatic and chromatic versions of the effect-must be fundamentally different phenomena. Ejima et al., in fact, using a (chromatic) light Ehrenstein pattern on a dark background, found that neon spreading also occurred when the luminance of the target lines was higher than the luminance of the external lines. Since this luminance sequence was considered ineffective for neon brightness spreading, it was concluded that the chromatic and achromatic effects must be mediated by separate mechanisms. The data reported in the present paper (Case 3) suggest that this conclusion is unwarranted. Note, by the way, that the transparency-based interpretation of neon spreading predicts that the best cases of neon spreading, for this particular luminance hierarchy, should be obtained when the luminance differences within the lattice (target vs. external lines) are as small as possible. For the achromatic effect, this prediction was confirmed in Experiment 2. For the chromatic effect, Ejima et al. found that the closer the target and external lines were in luminance, the stronger the neon spreading was. Again, this does not substantiate the notion that the chromatic and achromatic effects must be governed by different mechanisms.

I expect one crucial objection: the effects seen in Cases 2 and 3 have, phenomenally, little in common with the familiar neon color spreading phenomenon. One tends to look for vagueness and luminescence and a misty veil, and one may find neither in figures in which the luminance of the target lines is not in the middle. It is, however, hard to see what relevance this objection might have. If we accept that the neon color spreading effect occurs when segments of an otherwise homogeneous lattice are changed in color, and that it consists of the impression of a slightly colored filter or light lying above or behind the lattice, we must accept that the effects seen in Cases 1, 2, and 3 are identical. Neon spreading has been considered a novel effect because it looks different from traditional color assimilation, and it has been considered a theoretically interesting effect because the conditions for its occurrence look different from those for traditional color assimilation. As I have tried to demonstrate, the only genuine difference between the two phenomena is that the illusory color mixes with the background in assimilation patterns, while it goes to a separate, see-through layer in neon spreading patterns. From this point of view, again, the effects seen in Cases 1,2, and 3 are identical.

One may still choose to label only the effect seen in Case 1 as "neon spreading," but this only leads as far as having to find new names for the effects seen in Cases 2 and 3 . Thus it may be more appropriate to think of different modes of color appearance within the same phenomenon rather than of different phenomena. Depending on the lightness relationships in the pattern, the light or dark cast generated by assimilation can be attributed to a transparent overlay (if the underlying contrast is reduced, Cases 1 and 2), or to the illumination (if the under- lying contrast is increased, Case 3). These alternative "interpretations" have different phenomenal consequences. The filter stands in front of the lattice, making the underlying structure "vague." The patch of light or shade is less clearly located in depth and may appear behind the lattice; it makes the underlying structure more conspicuous, rather than vague. ${ }^{3}$

All these different perceptual organizations do have something in common, however: the phenomenal splitting of the illusorily altered color into two overlapping colors. It has been claimed that transparency can occur with or without color scissioning (Beck et al., 1984), in the sense that the colors in the overlapping region may look wrong. Although it is true that transparency is often seen where it is physically impossible, and that the observer's puzzlement can do very little to change the way things look, I would not say that color scissioning is ever impaired. It might be stated that color scissioning is here anomalous or paradoxical, but to perceive transparency means to perceive two colors simultaneously, one behind the other-however unrealistic the mixture may appear. In any case, color scissioning was always reported, along with transparency, in the experiments described here: the illusory tinge was ascribed either to a superimposed film (scission between surface colors) or to a cast light or shadow (scission between surface and illumination colors).

When simple assimilation is reported, on the contrary, there is no color scissioning within the target region: its color goes either entirely to the figure (in the front-square percept) or entirely to the background (in the front-hole percept). In these cases, perceived coplanarity between the inducing elements and the region where the spreading of color takes place is clearly crucial: it allows the induced color to mix with the region's color, thus taking on the phenomenal attributes of Bezold-like assimilation. Incidentally, note that this process has the side result of decreasing color differences within coplanar regions (here, either the bicolored square or the bicolored background) and increasing color differences between noncoplanar regions (here, either the square and its background or the frame and the background behind the hole).

Minimization of color unevenness within figures is, however, much more effective when transparency-in its broadest sense-is allowed. A superimposed filter neutralizes, at the same time, the color difference within the concentric-circle patterns (which become one color and are partly seen through) and the color difference within the background (which becomes, again, one color and is partly seen through); the color of the filter is always perceived as uniform throughout. Cast shadows and light spots are also seen as homogeneous and independent films separable from the surface on which they lie. It should be noted that, in the experiments reported above, "anomalous" transparency (transparency without color constancy) was never reported; that is, the color of the overlaid part of the lattice (the concentric-circle patterns) was invariably seen as the same as the color of the rest of the 
lattice. This was also true in the absence of transparency, where color constancy within the lattice was preserved through its amodal completion behind the illusory square.

The finding that neon spreading was observed in all, and only in, cases that were consistent with the occurrence of perceptual transparency in the assimilation region supports the view (Bressan, 1993) that the neon color spreading phenomenon depends uniquely on the occurrence of perceptual transparency. (Note that although it is trivial that neon spreading should entail transparency, because of the very way in which neon spreading is defined, it is not trivial that transparency in assimilation patterns should, regardless of the lightness hierarchy, entail neon spreading.) In particular, this means that neon spreading needs no special figural or luminance prerequisites other than those necessary for perceiving transparency. In a more practical vein, this implies that, perhaps, while there must be mechanisms responsible for ordinary color spreading (assimilation) and for ordinary color scissioning (phenomenal transparency), we should not look for other mechanisms that are specifically responsible for neon color spreading.

\section{CONCLUSIONS}

The experiments reported here show that van Tuijl and de Weert's (1979) luminance conditions for the occurrence of neon spreading should be considered exceedingly conservative. Neon spreading also occurs if the luminance of the target lines, rather than being between the luminances of the external lines and of the background, is either the highest or the lowest. When the target and external lines do not have the same contrast sign with respect to the background, the neon effect is easily obtained only with low-contrast target lines. When the target and external lines have the same contrast sign, the closer they are in luminance (i.e., the poorer the cues for a perceptual segregation of the lines are), the stronger the effect is. This result supports the widespread view that, for neon spreading to become possible, target and external lines must be seen as parts of the same structure (see van Tuijl $\&$ de Weert, 1979; see also Redies \& Spillmann, 1981). Note that only if they are seen as parts of the same structure can the deviation in color be attributed to a modifying agent (transparent matter or light).

If one likes to look at things from a functional angle, the outcome of this kind of scene organization makes much sense: despite local variations in color, regions bounded by aligned contours segregate together (i.e., go to the same object), and local variations are, wherever possible, discarded (i.e., the intrinsic color of the object tends to look uniform). Thus, the need to see unitary objects in a visual world where occlusion and illumination edges are ubiquitous seems to be the common denominator of transparency effects.

Although the phenomenon and mechanisms of color assimilation and the way in which the overall organization of a scene affects the mode of color appearance are both very interesting issues, neon spreading does not seem to convey any extra relevance. A neon spreading pattern appears to be very much like an ordinary four-surface transparency configuration, where the background and the part of the filter lying on it are very similar in lightness. Not surprisingly, when the illusorily colored subjective figure is replaced by a very slightly colored "real" figure, the effect is in all respects perceptually identical to neon spreading, if a little stronger (Bressan, unpublished data). Thus, the issue is not the peculiar phenomenal quality of the effect, but rather where the color comes from. The notion that it must come from somewhere other than where ordinary assimilation color comes from needs to be demonstrated. In the meantime, neon color spreading may not look as interesting as one might have thought; and it certainly appears to require no special allowances within models of color and form perception.

\section{REFERENCES}

BECK, J., IVRY, R. (1988). On the role of figural organization in perceptual transparency. Perception \& Psychophysics, 44 585-594.

Bfck, J., Prazdny, K.. \& IVRY, R. (1984). The perception of transparency with achromatic colors. Perception \& Psychophysics, 35. 407-422.

Bressan, P. (1993). Neon color spreading with and without its figural prerequisites. Perception, 22, 353-361.

de Weert, C. M. M., \& van Kruysbergen, A. W. H. (1987). Subjective contour strength and perceptual superimposition: Transparency as a special case. In S. Petry \& G. E. Meyer (Eds.), The perception of illusory contours (pp. 165-170). New York: Springer-Verlag.

Ejima, Y., Redies, C., Takahashi, S., A Akita, M. (1984). The neon color effect in the Ehrenstein pattern: Dependence on wavelength and illuminance. Vision Research, 24, 1719-1726.

MASIN, S. C. (1978). A contribution to the theory of perceptual scission in phenomenal transparency. Italian Jourmal of Psychology, $\mathbf{5}$, 171-191.

Metelli, F. (1974a). Achromatic color conditions in the perception of transparency. In R. B. MacLeod \& H. L. Pick (Eds.), Perception: Essays in honor of J. J. Gibson (pp. 95-116). Ithaca, NY: Comell University Press.

MetelLI, F. (1974b, April). The perception of transparency. Scientific American, 230, pp. 90-98.

METELLI, F. (1975). On the visual perception of transparency. In G. B. Flores D'Arcais (Ed.), Studies in perception (pp. 445-487). Milan: Martello.

MetelLI, F. (1985). Stimulation and perception of transparency. Psychological Research, 47, 185-202.

Metelli, F., Masin, S. C., Manganelli, A. M. (1980). Partial transparency. Atti dell'Accademia Patavina di Scienze, Lettere ed Arti, 92, 115-169.

Meyer, G. E., \& Dougherty, T. (1987). Effects of flicker-induced depth on chromatic subjective contours. Journal of Experimental Psychology: Human Perception \& Performance, 13, 353-360.

Nakayama, K., Shimojo, S., \& Ramachandran, V. S. (1990). Transparency: Relation to depth, subjective contours, luminance, and neon color spreading. Perception, 19, 497-513.

Redies, C., \& SpillmanN, L. (1981). The neon color effect in the Ehrenstein illusion. Perception, 10, 667-681.

VAN Tuis, H. F. J. M. (1975). A new visual illusion: Neonlike color spreading and complementary color induction between subjective contours. Acta Psychologica, 39, 441-445.

VAN TuiJL, H. F. J. M., \& DE WeERT, C. M. M. (1979). Sensory conditions for the occurrence of the neon spreading illusion. Perception, $8,211-215$

Van Tuisl, H. F. J. M., \& LeeuWenberg, E. L. J. (1979). Neon color 
spreading and structural information measures. Perception \& Psychophysics, 25, 269-284.

VARIN, D. (1971). Fenomeni di contrasto e diffusione cromatica nell'organizzazione spaziale del campo percettivo. Rivista di Psicologia, $\mathbf{6 5}, 101-128$.

\section{NOTES}

1. A possible objection to the critical role of transparency in the neon spreading phenomenon is the following. Not necessarily (and this is especially true in very simple neon spreading patterns, like the modified Ehrenstein cross), the color of the "overlaid" part of the structure is seen as the same as the color of the rest of the structure. In other words, when a red cross is embedded in a black Ehrenstein figure on a white background, the cross may look red rather than black, though seen through a red filter. The answer to this difficulty is that perception of transparency can occur without color constancy. This phenomenon is accounted for in Metelli's model, where it is called anomalous transparency (see Masin, 1978; Metelli, Masin, \& Manganelli, 1980), and it has been noticed independently by other authors (Beck, Prazdny, \& Ivry, 1984). The failure of color constancy in transparency might be related to transparency's being often unbalanced-that is, to the fact that the part of the filter lying on the figure may appear to have a lower, or higher, degree of transparency than the part of the filter lying on the background; in the extreme case, either part of the filter may look opaque (partial transparency). The uniformity of the color of the transparent layer (e.g., pinkish in the Ehrenstein cross case), which is a necessary condition for transparency, is observed, however, in both balanced and unbalanced transparency; and unbalanced transparency conditions do not hinder the perception of transparency.

2. The same line of reasoning also holds if luminances (the physical variables), rather than lightnesses (the perceptual variables), are considered. In this case $p=a$; the Magnitude Restriction $|a-b|>|p-q|$ can be rewritten as $|a-b|>|a-\mathrm{q}|$, and the order restriction ( $a>$ b) $\Leftrightarrow(p>q)$ and $(a<b) \Leftrightarrow(p<q)$ can be rewritten as $(a>$ $b) \Leftrightarrow(a>q)$ and $(a<b) \Leftrightarrow(a<q)$. The only difference (relative to the lightness version) is that in Case 3 ( $q>b>p=a$ and reverse) the magnitude restriction is never fulfilled, because the difference between $a$ and $b$ is always smaller than the difference between $a$ and $q$. It should be stressed that the fact that the physically different shades of gray are only three instead of four $(p=a)$ does not represent an obstacle to the application of the model (Metelli, 1975).

3 . Notice that cases like these, in which the subjective square is seen behind the concentric patterns, represent instances of illusory contours not associated with occlusion. It seems that these cases ought to have a theoretical weight within theories of illusory contours.

(Manuscript received April 23, 1992 ; revision accepted for publication December 4, 1992.) 